\title{
A 2D channel-clogging biofilm model
}

\author{
H. F. Winstanley ${ }^{1}$, M. Chapwanya ${ }^{2}$, A. C. Fowler ${ }^{1}$, and S. B. G. O'Brien ${ }^{1}$ \\ ${ }^{1}$ MACSI, Department of Mathematics and Statistics, University of Limerick, Limerick, Republic of Ireland \\ ${ }^{2}$ Department of Mathematics and Applied Mathematics, University of Pretoria, Pretoria 0002, South Africa
}

August 29, 2014

\begin{abstract}
We present a model of biofilm growth in a long channel where the biomass is assumed to have the rheology of a viscous polymer solution. We examine the competition between growth and erosion-like surface detachment due to the flow. A particular focus of our investigation is the effect of the biofilm growth on the fluid flow in the pores, and the issue of whether biomass can grow sufficiently to shut off fluid flow through the pores, thus clogging the pore space. Net biofilm growth is coupled along the pore length via flow rate and nutrient transport in the pore flow. Our 2D model extends existing results on stability of $1 \mathrm{D}$ steady state biofilm thicknesses to show that, in the case of flows driven by a fixed pressure drop, full clogging of the pore can indeed happen in certain cases dependent on the functional form of the detachment term.
\end{abstract}

Keywords: biofilm, detachment, clogging, channel, mathematical model.

\section{Introduction}

Biofilm appears to be the preferred form of life for bacteria in many environments and conditions: bacterial cells embed themselves in a slime matrix of secreted polysaccharides (EPS) and water, usually adhered to a surface [5]. Biofilm is practically ubiquitous on Earth, wherever there is water. Biofilm-forming bacteria subsist and often flourish across an astonishing diversity of environments, requiring only a minimum sufficient supply of essential nutrient elements in viable form and an exploitable form of energy (usually chemical or light) [27].

In general, water flow is required to provide the nutrient supply and prevent the buildup of metabolic products. In groundwater flows, microbial action transforms diverse organic contaminants into less harmful products, constituting the primary mechanism for groundwater purification in aquifers.

Where biofilm grows in a constrained space, whether in the pore spaces of soil and aquifer porous materials or in industrial or physiological tubes, there is potentially a feedback interaction between the biofilm and the water flow. Constriction of flow width can increase flow resistance and lead to reduced flow; reduced flow reduces the supply of nutrients along 
the flow path and thereby limits growth downstream. Furthermore, flow-induced shear stress can cause loss of biofilm either as surface detachment or as catastrophic sloughing at any point along the flow path.

In many settings biofilm growth is insufficient to affect flow appreciably, either because flow channels are wide, or because the biofilm growth is limited by nutrient supply, chemical disinfection, predation, or detachment/sloughing. In settings where the growth is sufficient to impinge on flow, the effect is termed clogging or bioclogging. Clogging in industrial and physiological applications generally has undesirable effects on process efficiency and health. In typical nutrient-poor groundwater conditions, biomass is sparsely distributed and has little effect on flow, but deliberate encouragement of excess growth blocking the pore space can be exploited in the form of 'biobarriers' so as to prevent (or slow) contaminated flow reaching a sensitive location $[2,6]$.

The focus of the present study is the competition between the factors of flow, nutrient supply, and detachment in determining biofilm growth. There is an extensive biofilm literature which has examined many aspects of this at the biofilm and pore scale, though it is clear that the detachment process remains poorly understood. Rittmann [17] suggested a power-law dependence of detachment on flow shear stress. Wanner and Gujer [26] suggested instead a quadratic dependence on the biofilm thickness (much repeated in the modelling literature, largely to ensure a steady state for numerical simulations), which received some support from arguments based on uniform probability of failure through the biofilm thickness in a 1D model by Stewart [20]. Kommedal and Bakke [12] fitted experimental data to a wide variety of functional forms for detachment, concluding that dependence on both shear stress and biofilm thickness/age are important. Duddu et al. [8] made a detailed numerical study of biofilm microcolony growth with shear-dependent and thickness-dependent detachment rates. Recent theoretical studies $[1,11]$ have examined the existence and stability of steady states in simple one-dimensional models of biofilm growth with loss terms due to decay or detachment.

These biofilm scale models contrast with models of pore clogging where the effect of biofilm growth on flow is examined at a larger scale. Early models using a basic geometric caricature of biofilm growth in the pore space of porous media [e.g. 4, 22] have largely given way to large numerical simulations of biomass growth in imaged or simulated geometries of porous medium samples [e.g. 14]. An alternative conceptual approach is provided by pore network models [e.g. 10, 21, 23] of clogging, which, out of computational necessity, are based on a lumped model of the flow-biofilm growth interaction in an individual pore.

The immediate aim of the present paper is to develop a model for a single channel and to examine the circumstances in which the interaction between flow and detachment can lead to a steady state solution. Most of the modelling literature on biofilm growth and pore clogging is predicated on the reasonable assumption that the biofilm thickness is limited, whether via a steady state balance between growth and loss terms, or via episodic sloughing events whose time-averaged effect is similar. Two recent theoretical studies have investigated the existence and stability of steady state biofilm thickness under the influence of thicknessand shear-dependent detachment terms $[1,11]$. While these models considered a strictly onedimensional biofilm, our model expands on their work by (a) taking into account nutrient transport in the pore water flow, and (b) including coupling along the pore length through the effect of biofilm thickness variation on flow resistance and nutrient supply. This longitudinal 
coupling varies according to the flow regime: if flow rate in the pore is specified, coupling is due to nutrient transport considerations alone; for flow driven by a specified pressure drop, a further nonlocal coupling exists as both the flow rate and nutrient supply are dependent on the biofilm thickness at all points along the pore. In applications such as porous media, where pores form an interconnected network with parallel flow paths, flow in an individual pore is effectively driven by a network-dependent combination of these extreme cases.

We use a similar single pore setting to that of van Noorden et al. [24], but where they undertook a theoretical exercise of upscaling by homogenisation, we seek to examine the behaviour of the pore scale model to gain insight into the form of the detachment term and the possibility of steady state biofilm distributions within the pore. This exercise may then provide a basis for comparison to experimental observations of biomass distribution so as to better constrain future modelling. The eventual purpose of this approach is to allow a parameterised lumped model of the individual pore for use in pore network models, where, in addition to any steady states of the single pore models, there is the possibility of limit cycle behaviour with 'flip-flop' switching between parallel flow paths due to alternating growth and net loss phases in each.

We consider a single pore channel saturated with nutrient-bearing water (see figure 1), which has biofilm attached symmetrically to the pore walls. Bacterial growth rate is assumed to be dependent on a single limiting nutrient, and we consider loss of the biofilm through first-order degradation in addition to detachment at the surface due to fluid shear stress. Our material model of the biofilm draws on previous work based on polymer solution theory $[28]$.

The outline of the paper is as follows: in section 2, we derive what we refer to as a microscopic model for the growth of a biofilm attached to the soil matrix in a single pore. The model is non-dimensionalised and simplified in section 3. In section 4 we provide some analytical insight before providing numerical solutions in section 5 . Conclusions are set out in section 6.

\section{Mathematical model}

We consider a single pore channel between parallel planar walls, having length $l$ and halfwidth $h$, with $\varepsilon=h / l \ll 1$. We define coordinate axes so that the full domain is $(x, z) \in$ $[0, l] \times[0,2 h]$. Biofilm grows on the pore walls to a thickness $s(x)$, and we assume symmetry across the pore centreline $z=h$ so that the model only considers the half-domain $(x, z) \in$ $[0, l] \times[0, h]$.

The unobstructed pore space $z \in(s, 2 h-s)$ is taken to be fully saturated and the longitudinal volume flux or flow rate is $q\left[\mathrm{~L}^{2} \mathrm{~T}^{-1}\right]$. The flow velocity is $\mathbf{u}=\left(u_{1}, u_{2}\right)$.

The biofilm is assumed to be predominantly extracellular slime matrix, a viscous solution of secreted bacterial extracellular polymeric substances (EPS). Its growth is dependent on the uptake of nutrient from the surrounding pore water, and the nutrient supply is thereby dependent on the flow. Biofilm growth constricts the pore space and increases flow resistance.

We ignore flow of water through the biofilm itself, despite its high water content, on the basis of recent results indicating that the effective permeability of biofilm is very low [25]. 


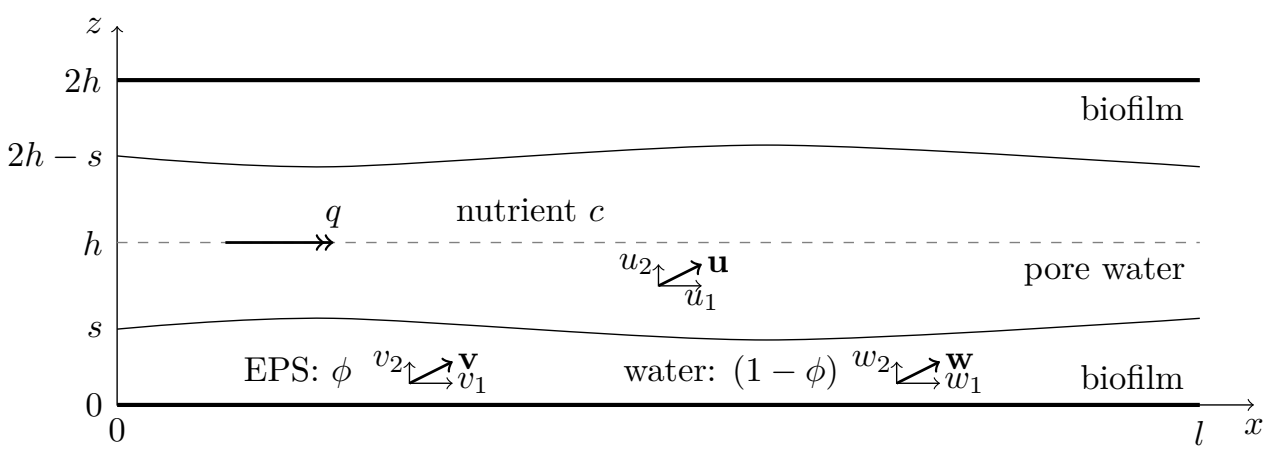

Figure 1: Definition sketch

\subsection{Biofilm model}

We start with the two phase biofilm growth model of Winstanley et al. [28], which describes the biofilm as a weak solution of EPS (volume fraction $\phi$, velocity $\mathbf{v}$, viscosity $\mu_{b}$ ) in water (volume fraction $(1-\phi)$, velocity $\mathbf{w}$ ). Growth is dependent on the local concentration of a single limiting nutrient $c$. The model can be written dimensionally as

$$
\begin{aligned}
\phi_{t}+\boldsymbol{\nabla} \cdot(\phi \mathbf{v}) & =g(\phi, c), & & {\left[T^{-1}\right] } \\
-\phi_{t}+\boldsymbol{\nabla} \cdot[(1-\phi) \mathbf{w}] & =-\varrho g(\phi, c), & & {\left[T^{-1}\right] } \\
\mu_{b} \boldsymbol{\nabla} \cdot\left[\phi\left(\boldsymbol{\nabla} \mathbf{v}+\boldsymbol{\nabla} \mathbf{v}^{T}\right)\right]-\zeta_{0} \phi(1-\phi)(\mathbf{v}-\mathbf{w})-\phi \boldsymbol{\nabla} p_{s} & =\mathbf{0}, & & {\left[M L^{-2} T^{-2}\right] } \\
\zeta_{0} \phi(1-\phi)(\mathbf{v}-\mathbf{w})-(1-\phi) \boldsymbol{\nabla} p & =\mathbf{0}, & & {\left[M L^{-2} T^{-2}\right] } \\
D \boldsymbol{\nabla} \cdot[(1-\phi) \boldsymbol{\nabla} c] & =r(\phi, c), & & {\left[M L^{-3} T^{-1}\right] }
\end{aligned}
$$

applying in the region $0<z<s(x)$ and $2 h-s(x)<z<2 h$. Equations $(2.1)_{1,2}$ represent conservation of EPS and water, while $(2.1)_{3,4}$ correspondingly expresses conservation of momentum. Conservation of nutrient is given by equation $(2.1)_{5} \cdot \varrho=\frac{\rho_{m}}{\rho_{l}}$ is the ratio of EPS and water densities, and $\zeta_{0}$ is an interphase drag coefficient. The EPS and water phase pressures $p_{s}$ and $p$, respectively, are related via

$$
p_{s}=p+\int_{\phi_{e q}}^{\phi} \frac{\Psi(\varphi) d \varphi}{\varphi},
$$

with an osmotic pressure term

$$
\Psi(\phi)=E_{L}\left(\ln (1-\phi)+\phi+\chi \phi^{2}\right)
$$

based on the long-chain limit of Flory-Huggins theory for polymer solutions. The value of the Flory parameter $\chi$ is assumed to be such that a non-zero EPS volume fraction $\phi_{e q}$ exists with $\Psi\left(\phi_{e q}\right)=0$. The nutrient uptake rate and biofilm (EPS) growth rate are given by $r(\phi, c)$ and $g(\phi, c)$, respectively.

Boundary conditions for this system are

$$
\begin{aligned}
& c=c_{s 0}, \quad \phi=\phi_{e q}, \quad p=\bar{p}(x), \quad \boldsymbol{\sigma} \cdot \mathbf{n}=\mathbf{0}, \quad \text { on } z=s(x), \\
& \frac{\partial c}{\partial z}=0, \quad \frac{\partial \phi}{\partial z}=0, \quad \frac{\partial p}{\partial z}=0, \quad \mathbf{v}=\mathbf{0}, \quad \mathbf{w} \cdot \mathbf{n}=0, \quad \text { on } z=0
\end{aligned}
$$


implying EPS adhesion to the impermeable, inert wall, and no stress and equilibrium swelling of the gel-like biofilm at the biofilm-water interface. The polymer phase stress tensor is given by

$$
\boldsymbol{\sigma}=\mu_{b}\left(\boldsymbol{\nabla} \mathbf{v}+\boldsymbol{\nabla} \mathbf{v}^{T}\right)-p_{s} \mathbf{I},
$$

and the biofilm surface evolves according to the kinematic condition,

$$
\frac{\partial s}{\partial t}=\left.(\mathbf{v}-E \mathbf{n}) \cdot \boldsymbol{\nabla}(z-s)\right|_{z=s},
$$

which includes a surface detachment rate $E\left[\mathrm{LT}^{-1}\right]$ as a non-negative erosive velocity normal to the biofilm surface. Using the scales

$$
\begin{aligned}
& c \sim c_{0}, \quad g \sim g_{0}, \quad \phi \sim \phi_{0}=\left(\frac{\zeta_{0} g_{0} D c_{0}}{r_{0} E_{L}}\right)^{1 / 3}, \quad s, \mathbf{x} \sim d=\left(\frac{D c_{0}}{r_{0}}\right)^{1 / 2}, \\
& t \sim t_{0}=\frac{\phi_{0}}{g_{0}}, \quad r \sim r_{0}, \quad \Psi, p \sim p_{0}=\frac{\zeta_{0} g_{0} D c_{0}}{r_{0}}, \quad \quad \mathbf{v}, \mathbf{w}, E \sim v_{0}=\frac{g_{0} d}{\phi_{0}},
\end{aligned}
$$

where $c_{0}, g_{0}$, and $r_{0}$ are representative values of nutrient concentration, EPS growth, and nutrient uptake, respectively. Winstanley et al. [28] used parameter estimation - and in particular the low volume fraction typically occupied by the EPS - to justify a simplified approximate model which may be written non-dimensionally as

$$
\begin{aligned}
& \phi_{t}+\nabla \cdot(\phi \mathbf{v})=g, \\
& \boldsymbol{\nabla} \cdot \mathbf{v}=-\boldsymbol{\nabla} \cdot\left(\frac{\nabla \Psi}{\phi}\right), \\
& \boldsymbol{\nabla} \times \boldsymbol{\nabla} \cdot\left[\phi\left(\boldsymbol{\nabla} \mathbf{v}+\boldsymbol{\nabla} \mathbf{v}^{T}\right)\right]=\mathbf{0}, \\
& \nabla^{2} c=r, \\
& \text { with } \Psi=\frac{1}{3} \phi^{2}(\phi-3 \lambda),
\end{aligned}
$$

where the right-hand term in $(2.1)_{2}$ for displacement of water by EPS growth and the viscous term in $(2.1)_{3}$ have been neglected as small. Equation $(2.7)_{2}$ comes from rearranging and taking the divergence of $(2.1)_{3}$, whilst equation $(2.7)_{3}$ only arises for models in greater than one spatial dimension, for which the inviscid limit in $(2.1)_{3}$ is singular. The polymer solution is in swelling equilibrium for $\phi=3 \lambda$, where the biofilm material parameter $\lambda=\left(\chi-\frac{1}{2}\right) / \phi_{0}$ is estimated as $\mathcal{O}(1)$. The limit $\nu=\left(9 \lambda^{3}\right)^{-1} \ll 1$ is a physically relevant one in which the EPS concentration is close to equilibrium throughout the biofilm. Substituting $\phi=3 \lambda(1+\nu \Phi)$ and rescaling $s, \mathbf{x}, \mathbf{v}, E \sim(3 \lambda)^{-1 / 2}$ and $r, g \sim 3 \lambda$, we have

$$
\begin{aligned}
\nu \Phi_{t}+\boldsymbol{\nabla} \cdot[(1+\nu \Phi) \mathbf{v}] & =g \\
\boldsymbol{\nabla} \cdot \mathbf{v} & =-\boldsymbol{\nabla} \cdot[(1+3 \nu \Phi) \boldsymbol{\nabla} \Phi], \\
\mathbf{0} & =\boldsymbol{\nabla} \times \boldsymbol{\nabla} \cdot\left[(1+\nu \Phi)\left(\boldsymbol{\nabla} \mathbf{v}+\boldsymbol{\nabla} \mathbf{v}^{T}\right)\right], \\
\nabla^{2} c & =r
\end{aligned}
$$


and thus for $\nu \ll 1$ this can be rearranged simply as

$$
\begin{aligned}
\nabla^{2} \Phi & =-g, \\
\nabla \cdot \mathbf{v} & =g, \\
\nabla \times \nabla^{2} \mathbf{v} & =\mathbf{0}, \\
\nabla^{2} c & =r .
\end{aligned}
$$

Boundary conditions are

$$
\begin{aligned}
& \frac{\partial c}{\partial z}=0, \quad \frac{\partial \Phi}{\partial z}=0, \quad \mathbf{v}=\mathbf{0} \quad \text { on } z=0, \\
& c=c_{s}=\frac{c_{s 0}}{c_{0}}, \quad \Phi=0, \text { no stress } \quad \text { on } z=s(x),
\end{aligned}
$$

with the kinematic condition (2.5) remaining unchanged.

\subsubsection{Quasi one dimensional biofilm model}

The additional simplification which we now make is to suppose that $s$ varies slowly with $x$ along the pore length so that a quasi one dimensional solution is appropriate. This is consistent with a common conceptual model of porous media in which pores are considered as long narrow channels [cf. 3], where in this case biofilm grows on the pore walls yet still lets flow through, and the biofilm is much thinner than it is long [see e.g. 19].

In one dimension, the system $(2.9),(2.10),(2.5)$ is simply

$$
\begin{aligned}
& \Phi^{\prime \prime}=-g, \quad \Phi^{\prime}(0)=0, \quad \Phi(s)=0, \\
& c^{\prime \prime}=r, \quad c^{\prime}(0)=0, \quad c(s)=c_{s}, \\
& \dot{s}=-\Phi^{\prime}(s)-E,
\end{aligned}
$$

where primes denote the spatial derivative with respect to $z$ and the overdot denotes the time derivative.

Typical dimensional forms for growth and uptake functions are

$$
\begin{aligned}
r(\phi, c) & =\frac{R \phi c}{K+c}, & & {\left[M L^{-3} T^{-1}\right] } \\
g(\phi, c) & =\frac{G \phi c}{K+c}-b \phi & & {\left[T^{-1}\right], }
\end{aligned}
$$

where $K$ is the half-saturation concentration for the nutrient. Appropriate scales for $c \lesssim K$ are $r_{0}=R \phi_{0} c_{0} / K$ and $g_{0}=G \phi_{0} c_{0} / K$, so that the nondimensional forms consistent with (2.9) are

$$
r=\frac{c}{1+c / \kappa}, \quad g=\frac{c}{1+c / \kappa}-\beta
$$

where we define

$$
\kappa=K / c_{0}, \quad \beta=\frac{b \kappa}{G} .
$$


The solution for $c$ is in general only available in implicit form, as

$$
\int_{c_{w}}^{c}\left[A(\hat{c})-A\left(c_{w}\right)\right]^{-1 / 2} d \hat{c}=\sqrt{2} z
$$

with

$$
A(c)=\kappa c-\kappa^{2} \ln \left(1+\frac{c}{\kappa}\right),
$$

and the pore wall concentration $c_{w}$ given by the quadrature

$$
\int_{c_{w}}^{c_{s}}\left[A(c)-A\left(c_{w}\right)\right]^{-1 / 2} d c=\sqrt{2} s .
$$

The reduced EPS content $\Phi$ and surface evolution $\dot{s}$ are then given by

$$
\begin{aligned}
\Phi & =c_{s}-c-\frac{1}{2} \beta\left(s^{2}-z^{2}\right), \\
\dot{s} & =c^{\prime}(s)-\beta s-E .
\end{aligned}
$$

An explicit solution for $c$ in $(2.11)_{2}$ is available for the case $c \ll \kappa$, which we can approximate with pseudolinear kinetics: $r=c, g=c-\beta$. The solution is then

$$
c=c_{s} \frac{\cosh z}{\cosh s},
$$

together with (2.18), so we see that for $c_{s}<\beta$ the biofilm thickness decreases regardless of any detachment term and the trivial steady state $s=0$ is stable. For $c_{s}>\beta$, the form of the detachment term $E$ governs the existence of non-trivial steady states and the stability of all steady states. $E \sim s^{n}$ as $s \rightarrow \infty$ for $n>1$ is sufficient to ensure the $s=0$ state is unstable so that biofilm may become established [11]. Similar conclusions arise for Monod kinetics, and indeed any kinetics for which $c^{\prime}(s)$ is monotonic increasing and $c^{\prime \prime}(s)<0$ [1]. Along the pore, depletion of nutrient from the flow ensures that any nontrivial steady state biofilm thickness must decrease monotonically in $x$.

Now reverting to dimensional quantities, we can write expressions for the evolution of the biofilm surface $s(t ; x)$ and for the nutrient uptake flux $J=\left.D \frac{\partial c}{\partial z}\right|_{s}$ in terms of the nutrient concentration $c_{s}$ at the biofilm surface in the case of Monod kinetics as

$$
\begin{array}{ll}
J=\sqrt{2 D R \phi_{e q}\left(c_{s}-c_{w}-K \ln \left[\frac{K+c_{s}}{K+c_{w}}\right]\right)} & {\left[M L^{-2} T^{-1}\right]} \\
\dot{s}=\frac{G}{R \phi_{e q}} J-b s-E, & {\left[L T^{-1}\right]}
\end{array}
$$

where $\phi_{e q}=3\left(\chi-\frac{1}{2}\right)$ is the equilibrium EPS volume fraction, and the pore wall concentration $c_{w}$ is given by the quadrature

$$
\sqrt{\frac{D}{R \phi_{e q}}} \int_{c_{w}}^{c_{s}}\left(c-c_{w}-K \ln \left[\frac{K+c}{K+c_{w}}\right]\right)^{-1 / 2} d c=\sqrt{2} s .
$$

For the case of the pseudolinear régime, (2.19) provides the simpler expression

$$
\begin{aligned}
J & =\frac{D c_{s}}{h} \alpha \tanh \left(\alpha \frac{s}{h}\right), & & {\left[M L^{-2} T^{-1}\right] } \\
\text { with } \quad \alpha^{2} & =\frac{h^{2} R \phi_{e q}}{D K}, & & {[-] }
\end{aligned}
$$


in place of $(2.20)_{1},(2.21)$, and the surface evolution is given as before by $(2.20)_{2}$. We can therefore consider the biofilm surface nutrient uptake flux to be $J=J\left(c_{s}, s\right)$.

We note that though the biofilm model is effectively one dimensional, the flow (and nutrient transport) in the pore is two dimensional and the two models are coupled along the pore length. The value of the nutrient concentration $c_{s}$ in the surface of the biofilm is thus specified by requiring concentration and flux continuity at the interface connecting the nutrient transport in the biofilm and the pore water (see figure 1).

\section{$2.2 \quad$ Pore flow model}

Water containing nutrients is driven through a pore by an imposed pressure gradient. For a long narrow pore with $\varepsilon=h / l \ll 1$, we assume the thin film flow approximation is valid (requiring $\varepsilon^{2} \operatorname{Re} \ll 1$, where $\operatorname{Re}=\rho U l / \mu$ for a suitable velocity scale $U$ ). The flow velocity $\mathbf{u}=\left(u_{1}, u_{2}\right)$ in $s(x)<z<h$ is given by

$$
\begin{aligned}
\mu u_{1 z z} & =p_{x}, \\
0 & =p_{z}, \\
u_{1 x}+u_{2 z} & =0,
\end{aligned}
$$

and we impose a no slip boundary condition at the biofilm surface, recognising that the water velocity within the biofilm is many times smaller than the free flow velocity as a result of the low biofilm growth velocity and low biofilm permeability. We assume symmetry at the pore centreline $z=h$ and note that the longitudinal pressure gradient $p_{x}$ varies with $x$ due to the variation of biofilm thickness $s(x)$. We can then write the flow field solution as

$$
\begin{aligned}
& u_{1}=\eta(z-s)(2 h-s-z), \\
& u_{2}=\frac{h-z}{h-s} s^{\prime} u_{1},
\end{aligned}
$$

with

$$
\eta=\frac{3 q}{4(h-s)^{3}} \cdot \quad\left[L^{-1} T^{-1}\right]
$$

We note $s^{\prime}=\frac{d s}{d x}(x)$ is small in the quasi-1D biofilm solution, and we consequently ignore the small transverse velocity component. The pressure is approximately uniform across the width of the pore and varies longitudinally as

$$
p(x)=p(0)-2 \mu \int_{0}^{x} \eta(\xi) d \xi . \quad\left[M L^{-1} T^{-2}\right]
$$

Hydrodynamic shear stress at the biofilm surface is

$$
\tau=\left.\mu \frac{\partial u_{1}}{\partial z}\right|_{z=s}=\frac{3 \mu q}{2(h-s)^{2}} .
$$

Combining (2.25) and (2.26) gives an expression relating the pressure difference $\Delta p=p(0)-$ $p(l)$ between inlet and outlet and the flow rate $q$,

$$
\Delta p=\frac{3 \mu q}{2 h^{3}} \int_{0}^{l}\left(1-\frac{s}{h}\right)^{-3} d x .
$$


The flow is then determined either by specifying some known $q$, or by specifying the total pressure drop $\Delta p$. In this latter case, we observe that these flow conditions introduce a nonlocal coupling due to the biofilm thickness $s(t ; x)$ at every point influencing the flow (and thence nutrient supply) throughout the pore length.

Within the pore, the nutrient is transported by advection and diffusion and we assume symmetry across the pore centreline $z=h$. We neglect time derivatives as our timescale of interest is that of biofilm growth which is much slower than diffusive nutrient equilibration across the narrow pore, so nutrient transport is given by the equation

$$
u_{1} c_{x}=D \nabla^{2} c \text { for } 0<x<l \text { and } s(x)<z<h .
$$

Boundary conditions are

$$
\begin{aligned}
& c_{z}=0 \quad \text { on } z=h, \quad c=c_{0} \quad \text { on } x=0 \text {, } \\
& D c_{z}=J(c, s) \quad \text { on } z=s, \quad c_{x}=0 \quad \text { on } x=l \text {, }
\end{aligned}
$$

where the biofilm nutrient flux $J(c, s)$ presumes nutrient concentration continuity across the interface, i.e. $c_{s}=\left.c\right|_{z=s^{+}}$.

\section{Non-dimensionalisation}

We non-dimensionalise the model (2.18), (2.20), (2.24)-(2.30) with scales

$$
\begin{aligned}
& x \sim l, \quad s, z \sim h, \quad t \sim \frac{\kappa}{G}, \quad E \sim \frac{h G}{\kappa}, \quad c, c_{s}, c_{w} \sim c_{0}, \\
& J \sim \frac{\alpha^{2} D c_{0}}{h}, \quad \Delta p \sim P, \quad q \sim q_{0}=\frac{2 h^{3} P}{3 \mu l}, \quad \tau \sim \varepsilon P,
\end{aligned}
$$

where $c_{0}$ is the inlet nutrient concentration, and we have based the flow scale $q_{0}$ on the flow rate in the biofilm-free pore, for which the centreline flow velocity is $U=\frac{3 q_{0}}{4 h}$.

The non-dimensional model is then

$$
\begin{aligned}
\dot{s} & =J-\beta s-E, \\
\varepsilon \mathrm{Pe} \frac{(z-s)(2-s-z)}{(1-s)^{3}} q c_{x} & =\left[\varepsilon^{2} c_{x x}\right]+c_{z z},
\end{aligned}
$$

where we define a pore Péclet number

$$
\mathrm{Pe}=\frac{U h}{D}=\frac{3 q_{0}}{4 D} .
$$

In addition to $\varepsilon \ll 1$, we assume $\varepsilon \ll$ Pe so that the dominant balance is between longitudinal advection and transverse diffusion, and consequently neglect the $\mathcal{O}\left(\varepsilon^{2}\right)$ longitudinal diffusion term in $(3.2)_{2}$.

Boundary conditions are

$$
\begin{array}{ll}
c=1 & \text { on } x=0, s<z<1, \\
c_{x}=0 & \text { on } x=1, s<z<1, \\
c_{z}=0 & \text { on } 0<x<1, z=1, \\
c_{z}=\alpha^{2} J(c, s) & \text { on } 0<x<1, z=s,
\end{array}
$$


and initial conditions $s=s_{0}(x)$ at $t=0$. Non-dimensional nutrient uptake flux for Monod kinetics is given by

$$
J=J\left(c_{s}, s\right)=\frac{\sqrt{2}}{\alpha}\left[A\left(c_{s}\right)-A\left(c_{w}\right)\right]^{1 / 2},
$$

with $c_{w}$ calculated from

$$
\sqrt{2} \alpha s=\int_{c_{w}}^{c_{s}}\left[A(c)-A\left(c_{w}\right)\right]^{-1 / 2} d c
$$

and $A(c)$ is given by (2.16). For pseudolinear kinetics, (3.5) and (3.6) are replaced by

$$
J\left(c_{s}, s\right)=\frac{c_{s}}{\alpha} \tanh (\alpha s) .
$$

Shear stress at the biofilm surface is

$$
\tau=\frac{q}{(1-s)^{2}}
$$

while flow rate and pressure drop are connected via

$$
q=\Delta p\left[\int_{0}^{1}(1-s)^{-3} d x\right]^{-1}
$$

so that in the case of flow driven by a specified pressure drop $\Delta p$ this relation introduces longitudinal nonlocal coupling. By contrast, in the case of fixed flow rate $q$ coupling arises

only in the downstream direction (when longitudinal diffusion is negligible) through nutrient transport in the pore flow.

\section{The influence of biofilm detachment}

The extent of pore clogging is determined by the biofilm surface evolution from $(3.2)_{1}$,

$$
\dot{s}=J\left(c_{s}, s\right)-\beta s-E .
$$

Typical forms of the biofilm detachment term in the literature [cf. 1, 11, 12] take the form

$$
E= \begin{cases}\theta s^{n} \tau^{m} & \text { for } s>0 \\ 0 & \text { for } s=0\end{cases}
$$

with constants $\theta, n, m \geq 0$ and common choices being $n=1,2$ and $0 \leq m \leq 1$.

We observe that $J\left(c_{s}, 0\right)=0$, and $\tau=\tau(s)$ so that $E=E(s)$. The choice $n=0$ therefore implies that $\dot{s} \leq 0$ close to $s=0$, so that the trivial solution $s=0$ is unconditionally stable and thin biofilm is unable to become established. Since we expect new biofilm to be able to form under suitable conditions, the $n=0$ case is excluded as unphysical. Choices $0<n<1$ can be similarly ruled out since they give $E(0)=0$ and $E^{\prime}(s) \rightarrow \infty$ as $s \rightarrow 0$, so the $s=0$ solution is also unconditionally stable. We therefore limit ourselves to $n \geq 1, m \geq 0$. The physical mechanisms by which the $s$-dependence arises are unclear, though potential explanations may involve biofilm stratification with greater cohesiveness towards the base $[7,18]$, or the size distribution of particles detached from the biofilm [cf. 20].

The trivial steady state $s=0$ is unstable/stable for $F(0) \gtrless 0$, where

$$
F(s)=\frac{d J}{d s}-\beta-E^{\prime} .
$$




\section{Fixed flow rate $q$}

In the case of a fixed flow rate $q$, we have $\tau=q(1-s)^{-2}$ and $E(s)=\theta s^{n} q^{m}(1-s)^{-2 m}$. We immediately see that $E(s) \rightarrow \infty$ as $s \rightarrow 1$, so the biofilm is unable to clog the channel entirely and when the $s=0$ state is unstable there is a partially clogged steady state $0<s<1$ which is stable. For $n \geq 1$, we have $E(0)=0$ and $E^{\prime}(0)=n \theta q^{m} s^{n-1}$, and the trivial steady state $s=0$ is unstable/stable if $\frac{d J}{d s}-\beta-n \theta q^{m} s^{n-1} \gtrless 0$. The biofilm can become established if growth is strong enough, and for the particular choice $n=1$ the stability is independent of flow conditions.

For pseudolinear kinetics (3.7) we have $\frac{\partial J}{\partial c_{s}}=0$ at $s=0$, so $\frac{d J}{d s}\left(c_{s}, 0\right)=\frac{\partial J}{\partial s}\left(c_{s}, 0\right)=c_{s}$ and the biofilm will decay to extinction if

$$
\text { [pseudolinear] } \quad F(0)=c_{s}-\beta-\delta_{1 n} \theta q^{m}<0, \quad \text { i.e. } \quad c_{s}<\beta+\delta_{1 n} \theta q^{m} \text {, }
$$

where $\delta_{1 n}=1$ for $n=1$ and $\delta_{1 n}=0$ otherwise. Within a pore channel under fixed flow conditions, nutrient depletion along the pore length means $c$ and $c_{s}$ decrease monotonically in $x$ and there may be a downstream position beyond which this criterion is satisfied and the biofilm is not viable.

For Monod kinetics, the simplest case is to approximate the situation of a pore in which uptake of nutrient along the pore length is small so that $c \approx c_{s} \approx 1$. In this case $(2.16)$ and (3.6) imply

$$
\sqrt{2} \alpha s \approx \frac{2}{A^{\prime / 2}}\left(c_{s}-c_{w}\right)^{1 / 2}
$$

and approximating $c=c_{s}+\mathcal{O}(\alpha)$ we have

$$
J \approx A^{\prime} s=\frac{c_{s} s}{1+c_{s} / \kappa}
$$

from (3.5). This situation is similar to the 1D models of Klapper [11] and Abbas et al. [1]. Using (4.6) in (4.3), we see that the biofilm will decay to extinction if

$$
\begin{aligned}
\text { [simplified Monod] } F(0) & =\frac{c_{s}}{1+c_{s} / \kappa}-\beta-\delta_{1 n} \theta q^{m}<0, \\
\text { i.e. } \quad c_{s} & <\frac{\beta+\delta_{1 n} \theta q^{m}}{1-\frac{1}{\kappa}\left(\beta-\delta_{1 n} \theta q^{m}\right)},
\end{aligned}
$$

where $\delta_{1 n}=1$ for $n=1$ and $\delta_{1 n}=0$ otherwise.

Conversely, when the trivial steady state is unstable, the convexity of $E(s)$ for fixed $q$ ensures that the nontrivial steady state in $0<s<1$ is unique and stable provided $\frac{\partial^{2} J}{\partial s^{2}} \leq 0$ in $0<s<1$.

\section{Fixed pressure drop $\Delta p$}

In the case of a fixed pressure drop $\Delta p$, the flow $q$ is dependent on the biofilm thickness $s$ throughout the pore via (3.9), so

$$
\tau=\frac{\Delta p}{(1-s)^{2} \int_{0}^{1}(1-s)^{-3} d x} .
$$


It is instructive first to consider the simple case of uniform $s$ in which

$$
\tau=\Delta p(1-s), \text { and } \quad E(s)=\theta s^{n} q^{m}(1-s)^{m} .
$$

We see that for $n \geq 1$ and $m>0, E(s)=0$ for both the biofilm-free and fully clogged cases $s=0,1$. Consequently, it appears fully clogged solutions $s \rightarrow 1$ may exist under certain conditions, and the existence and stability of partially clogged steady states $0<s<1$ will be dependent on the forms of $J$ and $E$.

The stability of the trivial steady state $s=0$ mirrors the fixed $q$ situation, being unstable for

$$
\begin{aligned}
\text { [pseudolinear] } & c_{s}<\beta+\delta_{1 n} \theta \Delta p^{m} \\
\text { [simplified Monod }] & c_{s}<\frac{\beta+\delta_{1 n} \theta \Delta p^{m}}{1-\frac{1}{\kappa}\left(\beta-\delta_{1 n} \theta \Delta p^{m}\right)} .
\end{aligned}
$$

If $s=0$ is unstable, nontrivial steady states $s>0$ must nonetheless exist if $\frac{\partial^{2} J}{\partial s^{2}} \leq 0$. However, these steady states may not be unique and steady states $s>1$ are not achievable as they imply full clogging and breakdown of the model. Abbas et al. [1] analysed a simple $1 \mathrm{D}$ model of a well-mixed bioreactor with approximate Monod kinetics (4.6) and $n=1$, $m=\frac{1}{2}$. They found that for small $\theta \Delta p^{m}<\frac{d J}{d s}-\beta$ a single nontrivial steady state existed, in addition to the unstable trivial equilibrium, and was stable, being limited by nutrient supply (through $\frac{\partial J}{\partial c_{s}}$ ) rather than detachment; full clogging did not occur. In at least part of the range $0<\frac{d J}{d s}-\beta<\delta_{1 n} \theta \Delta p^{m}$ two nontrivial equilibria, one stable and the other unstable, coexisted with the stable trivial steady state $s=0$.

In the $2 \mathrm{D}$ case, nutrient depletion along the pore length means $c$ and $c_{s}$ decrease monotonically in $x$, and we therefore might expect that $J$ will similarly decrease in $x$. Presuming nutrient conditions at inlet are such that the biofilm is viable $F(0)>0$, the anticipated longterm stable steady state is one in which the biofilm thickness is greatest at the inlet $x=0$ and decays monotonically with increasing $x$. Nutrient depletion along the channel length may be such that no stable solution other than $s=0$ exists in a downstream region ('death zone') beyond a certain location.

The 2D case does not admit a straightforward analysis of nontrivial steady states as for the 1D models, but we see that $J$ need not be monotonically decreasing in $x$ : $\frac{d J}{d x}=$ $\frac{\partial J}{\partial c_{s}} \frac{d c_{s}}{d x}+\frac{\partial J}{\partial s} \frac{d s}{d x}>0$ if

$$
\begin{aligned}
\text { [pseudolinear] } & s_{x}>-\frac{1}{\alpha c_{s}} \frac{d c_{s}}{d x} \sinh (\alpha s) \cosh (\alpha s), \\
\text { [simplified Monod] } & s_{x}>-\frac{d c_{s}}{d x} \frac{s}{c_{s}\left(1+c_{s} / \kappa\right)} .
\end{aligned}
$$

These conditions are only achievable from nonuniform initial conditions, so we investigate their stability numerically.

A significant question to consider is whether a fully clogged steady state is possible. Nutrient depletion appears to prevent full clogging in the well-mixed reactor model [1], but in the 2D advective transport model nutrient is significantly depleted from the flow only downstream of the inflow (where effectively $c_{s}=1$ ) and therefore does not exercise the 


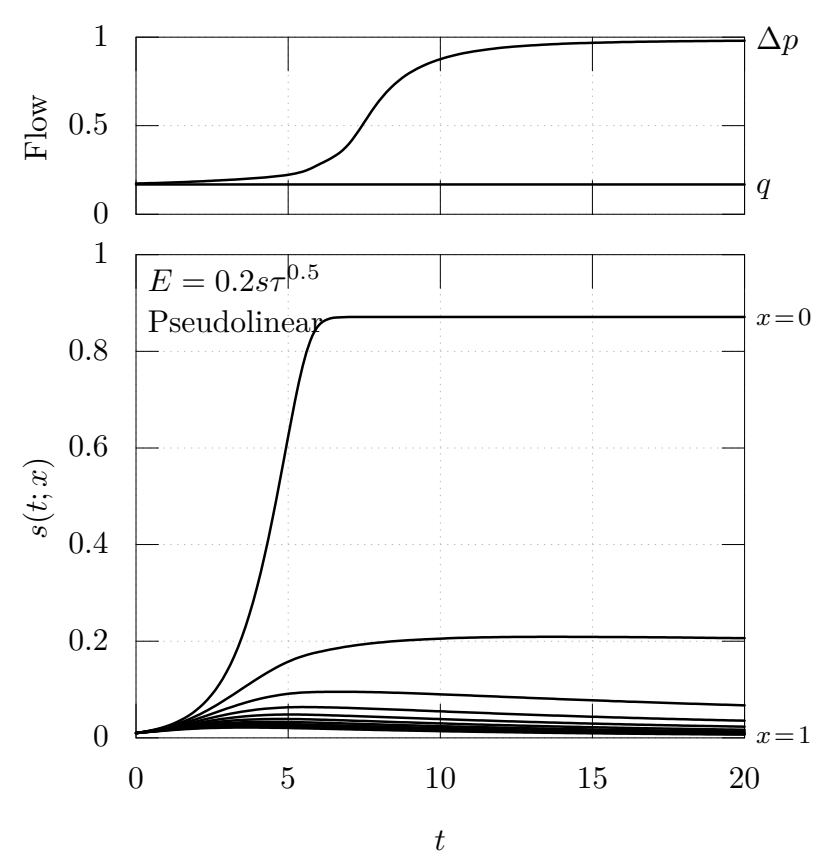

(a) Fixed $q$

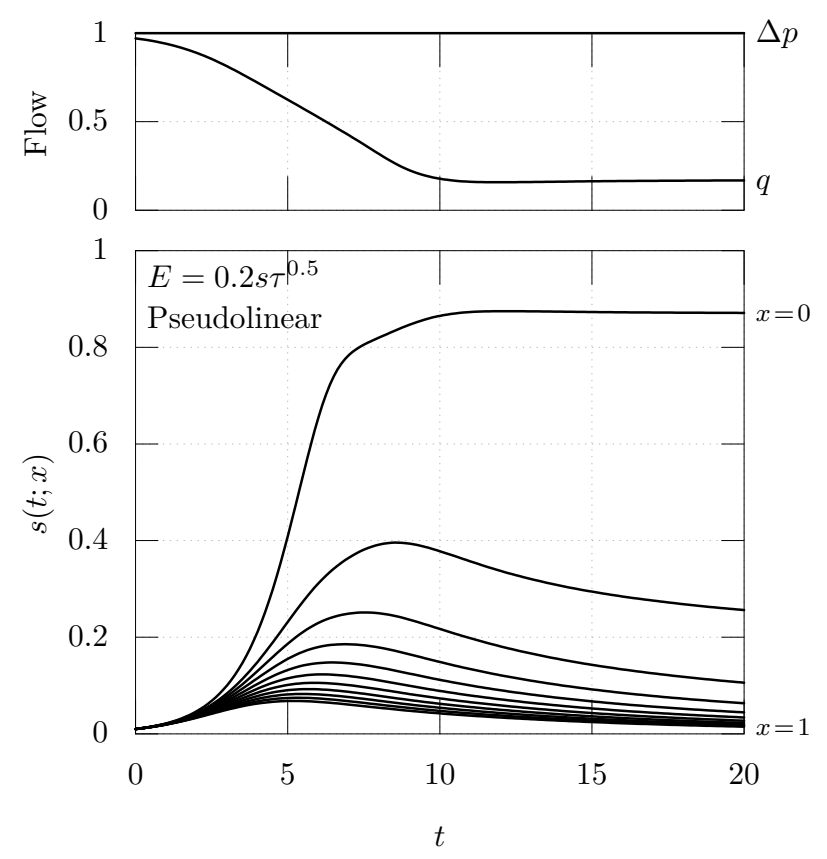

(b) Fixed $\Delta p$

Figure 2: Evolution of flow rate $q$, pressure drop $\Delta p$, and biofilm thickness $s(t ; x)$ for $x=$ $0,0.1, \ldots, 1$ with pseudolinear uptake kinetics. Initial condition $s_{0}(x)=0.01$.

same limitation on clogging at inflow. If the biofilm approaches full clogging at the inflow, downstream nutrient depletion will suppress downstream growth and increasingly skew the biofilm thickness towards the inlet. It is unclear in this case whether the nonlocal coupling of shear stress provides a mechanism by which full clogging can be avoided: from (4.8) we see that a biofilm of the form $1-s \sim x^{a}$ as $x \rightarrow 0$ with $0<a<\frac{1}{3}$ would ensure that $E \rightarrow \infty$ locally as $s \rightarrow 1$ and prevent full clogging, though the attainability of this scenario is uncertain.

If full clogging proceeds, flow and nutrient supply cease and the model breaks down. In reality, longitudinal diffusion of nutrient from the upstream end may support a biofilm plug whose extent and mechanical properties will determine if it can withstand the applied pressure difference or if structural failure will reinstate pore flow and restart the process.

\section{$5 \quad$ Numerical simulations}

The derived scaled model is two-dimensional on a fixed region $(x, z) \in[0,1] \times[0,1]$. However, this region is subdivided by a moving interface, $z=s$ into the pore region and the biofilm region. While the biofilm model is effectively $1 \mathrm{D}$ and numerical methods can be implemented directly, the pore flow model is more complicated and we consider transforming the region $(x, z) \in[0,1] \times[s(x), 1]$ into a unit square, $(\xi, \eta) \in[0,1] \times[0,1]$ by setting

$$
\xi=x, \quad \eta=\frac{z-s}{1-s} .
$$




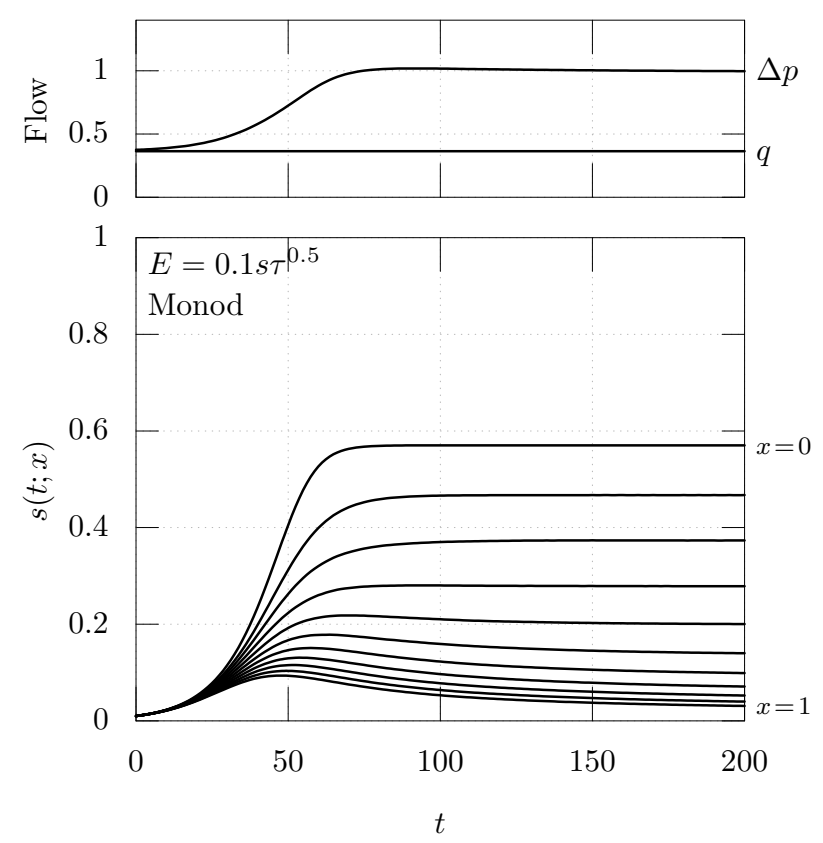

(a) Fixed $q$

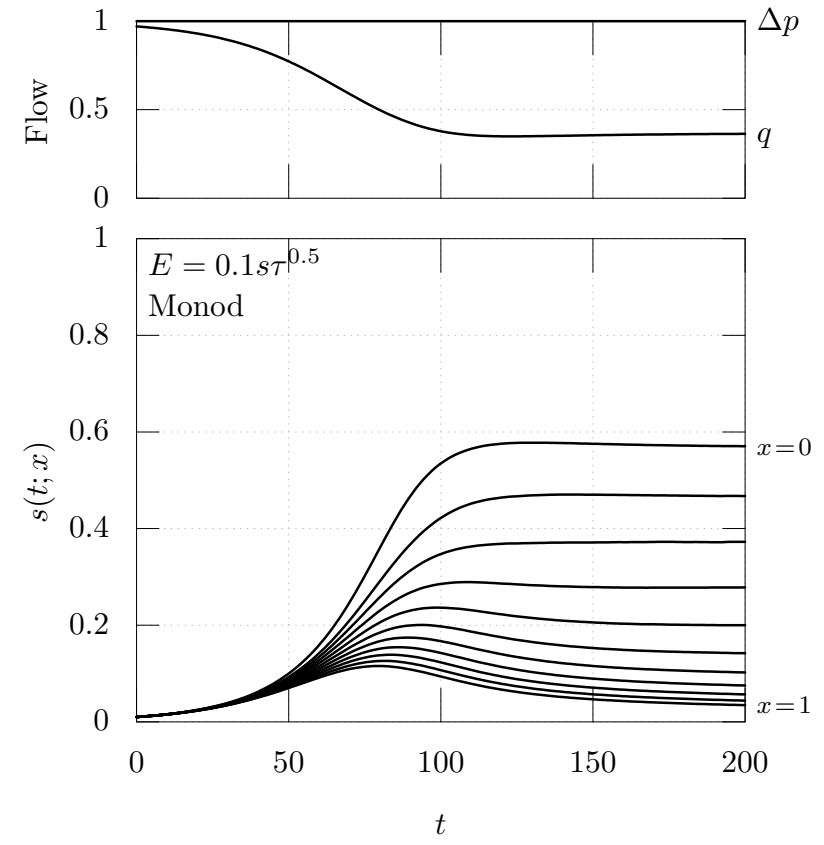

(b) Fixed $\Delta p$

Figure 3: Evolution of flow rate $q$, pressure drop $\Delta p$, and biofilm thickness $s(t ; x)$ for $x=$ $0,0.1, \ldots, 1$ with Monod uptake kinetics. Initial condition $s_{0}(x)=0.01$.

The transformed model becomes

$$
\begin{aligned}
\dot{s} & =J-\beta s-\theta s^{n}\left[\frac{q}{(1-s)^{2}}\right]^{m}, \\
\varepsilon \operatorname{Pe}(2-\eta) \eta(1-s) q c_{\xi} & =c_{\eta \eta}+\varepsilon \operatorname{Pe}(2-\eta)(1-\eta) \eta q s_{\xi} c_{\eta},
\end{aligned}
$$

with $q$ given in the fixed $\Delta p$ case by

$$
q=\Delta p\left[\int_{0}^{1}(1-s)^{-3} d \xi\right]^{-1} .
$$

Boundary conditions are specified as

$$
\begin{array}{ll}
c=1 & \text { on } \xi=0, \\
c_{\eta}=0 & \text { on } \eta=1, \\
c_{\eta}=\alpha^{2}(1-s) J & \text { on } \eta=0, \\
c=c_{s}, & \text { on } \eta=0,
\end{array}
$$

and the biofilm nutrient flux term $J=J\left(c_{s}, s\right)$ remains unchanged. We implement numerical solutions in MATLAB using the method of lines to solve both the ODE system (5.2) 1 with suitable initial condition $s(0 ; x)=s_{0}(x)$ and the inner loop $(5.2)_{2},(5.4)$ as an index-1 DAE system in $\xi$. The quadrature (3.5),(3.6) for the Monod case is computed by Newton iteration of a finite difference scheme.

For illustrative purposes we choose typical parameter values shown in table 1. 


\begin{tabular}{lclc}
\hline Parameter & Symbol & Typical value & Source \\
\hline pore half-width & $h$ & $50 \mu \mathrm{m}$ & {$[9]$} \\
pore length & $l$ & $600 \mu \mathrm{m}$ & {$[9]$} \\
pore pressure drop & $P$ & $0.1 \mathrm{~Pa}$ & {$[9]$} \\
water viscosity & $\mu$ & $10^{-3} \mathrm{Pas}$ & \\
inlet oxygen concentration & $c_{0}$ & $10^{-3} \mathrm{~kg} \mathrm{~m}^{-3}$ & {$[28]$} \\
diffusion coefficient & $D$ & $2 \times 10^{-9} \mathrm{~m}^{2} \mathrm{~s}^{-1}$ & \\
EPS volume fraction & $\phi_{e q}$ & 0.02 & {$[28]$} \\
growth coefficient & $G$ & $1.3 \mathrm{day}^{-1}$ & {$[28]$} \\
Monod constant & $K$ & $3.5 \times 10^{-4} \mathrm{~kg} \mathrm{~m}^{-3}$ & {$[28]$} \\
decay constant & $b$ & $0.12 \mathrm{day}^{-1}$ & {$[9]$} \\
uptake coefficient & $R$ & $2.6 \times 10^{3} \mathrm{~kg} \mathrm{~m}^{-3} \mathrm{day}^{-1}$ & {$[28]$} \\
\hline Biofilm nutrient utilisation & $\alpha$ & 1.5 & \\
Decay parameter & $\beta$ & $3.2 \times 10^{-2}$ & \\
Monod parameter & $\kappa$ & 0.35 & \\
Pore aspect ratio & $\varepsilon$ & $8.3 \times 10^{-2}$ & \\
Transverse Péclet number & Pe & 5.2 & \\
\hline
\end{tabular}

Table 1: Typical parameter values.

Typical solutions for detachment $n=1, m=\frac{1}{2}$ are shown in figures 2 (pseudolinear) and 3 (Monod) as profiles $s\left(t ; x_{i}\right)$ against $t$ at various positions $x_{i}$. In the solutions for fixed flow rate (a), $q$ is set so as to coincide with the steady state flow rate of the fixed pressure drop solutions (b) in which we set $\Delta p=1$. These solutions start with a uniform initial biofilm distribution and eventually evolve to a steady state in which the biofilm persists only in a region close to the inflow. The steady state is achieved very slowly, particularly at the downstream end of the channel, due to non-local negative feedback from biofilm decay partially alleviating nutrient limitation in other regions. This is most evident in the comparison of figure 2 (a) and (b). For fixed $q$, feedback is downstream-only, via nutrient depletion in the flow: at $x=0$ pore constriction due to growth yields to rapid onset of detachment, while nutrient depletion leads to decay (to $s=0$ stable) in an expanding downstream region. For fixed $\Delta p$, additional feedback from flow resistance acts both upstream and downstream: faster initial flow rate causes higher initial growth rate throughout the channel length, but the decrease in flow rate as the pore is constricted has the effect of smoothing the onset of detachment limitation at $x=0$ and causing a more rapid onset of decay due to nutrient depletion downstream (i.e. expansion of the death zone). Eventual steady states for the two flow regimes are indistinguishable.

In figure 3, differences between the two flow regimes are less evident as Monod growth rate saturation severely restricts the initial downstream growth overshoot. The fixed pressure drop solution evolves more slowly than the fixed flow solution, presumably due to the greater flow rate in the initial stages increasing the detachment rate.

Numerical experimentation confirms that the choice $n=1$ is qualitatively different from $n>1$ in that the stability of the trivial steady state is flow-dependent only when $n=1$. For $n>1, m>0$, simulations with viable nutrient conditions $\frac{d J}{d s}>\beta$ at inflow produced nontrivial steady states for all values of $\theta$. However, for $n=1$ larger values of $\theta$ render the 

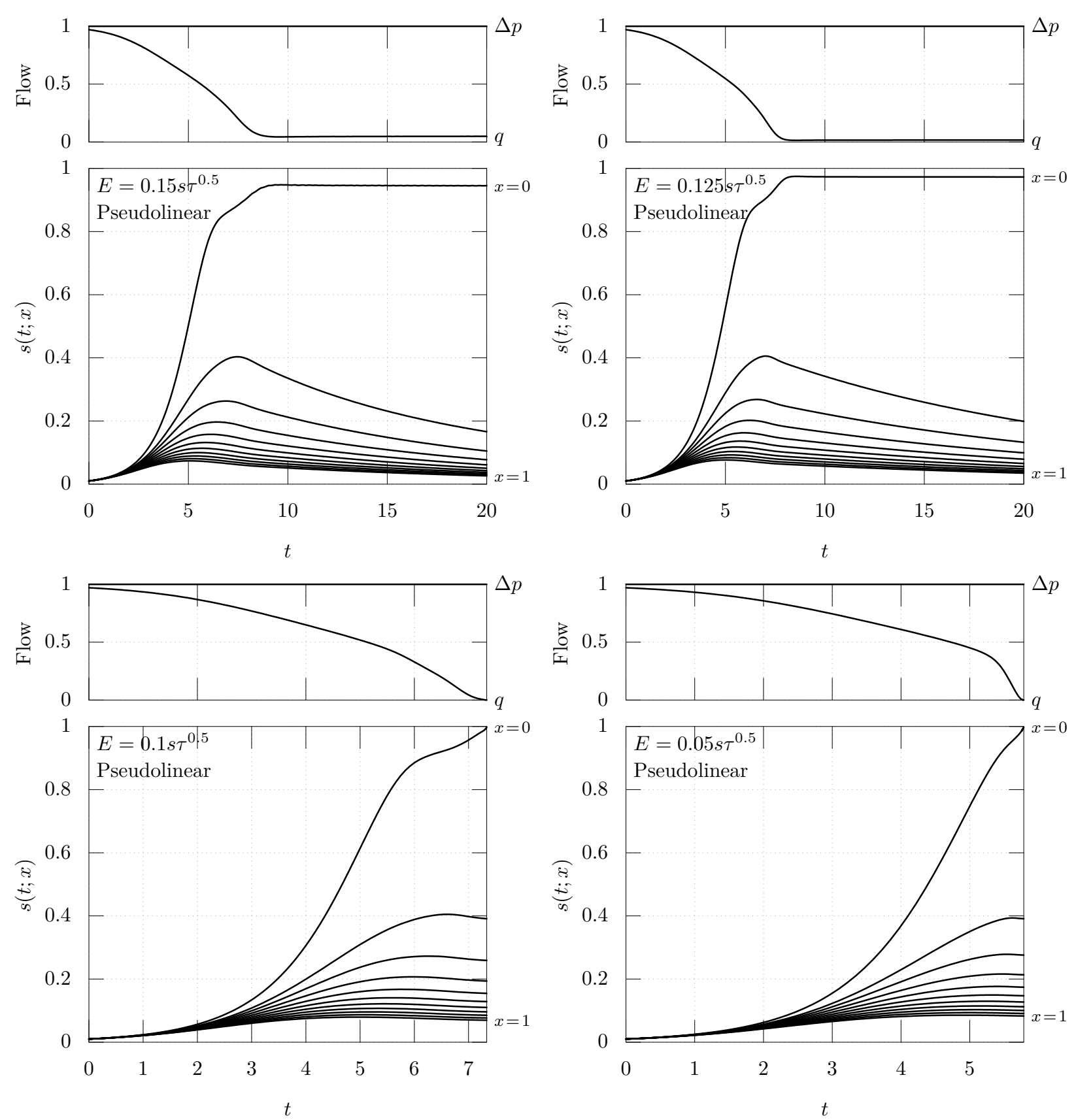

Figure 4: Evolution of flow rate $q$, pressure drop $\Delta p$, and biofilm thickness $s(t ; x)$ for $x=$ $0,0.1, \ldots, 1$ with pseudolinear uptake kinetics. Initial condition $s_{0}(x)=0.01$. 


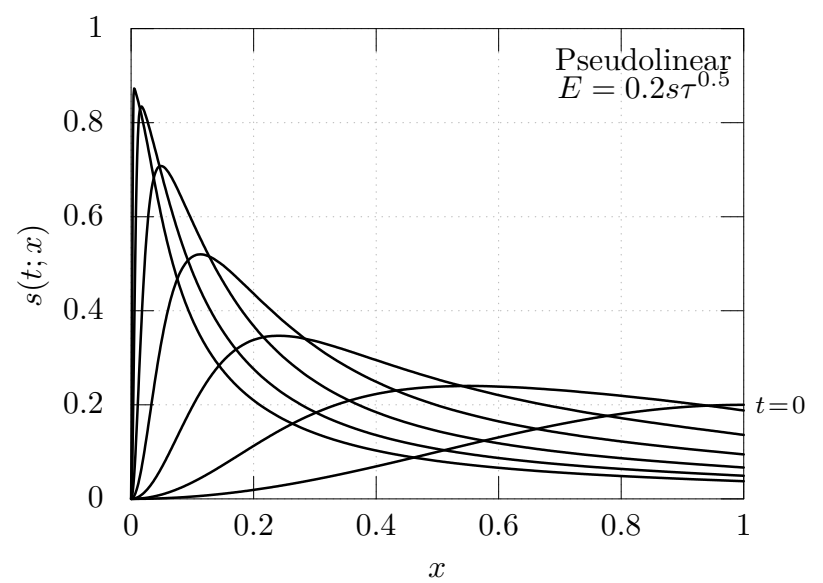

Figure 5: Evolution of flow rate $q$, pressure drop $\Delta p$, and biofilm thickness $s(t ; x)$ for $t=$ $0,2.5, \ldots, 15$ with pseudolinear uptake kinetics. Initial condition $s_{0}(x)=0.1(1-\cos \pi x)$.

trivial steady state stable at the inflow, such that the saturation-limited Monod kinetics case in figure 3 required a much smaller value of $\theta$ than the pseudolinear case of figure 2 in order to produce a nontrivial steady state.

For $n=1$, the inequalities $(4.4),(4.7),(4.10)$ for viability of the biofilm imply a threshold value $\theta=\theta_{\text {crit }}$ above which biofilm cannot persist at the inflow $x=0$ to be tested against the numerical model. For pseudolinear kinetics these threshold values apply accurately. For Monod kinetics, the approximated threshold overestimates the numerically-estimated threshold ( 0.23 vs. 0.15 for $n=1, m=\frac{1}{2}$ ).

We investigate numerically the question of whether the biofilm is able to clog the full pore width when flow is driven by a fixed pressure drop. Results shown in figure 4 suggest that full clogging is indeed possible for sufficiently small $\theta$, in contrast to the well-mixed bioreactor model of Abbas et al. [1]. Clearly the numerical solution becomes ill-conditioned in the approach to the limit $s \rightarrow 1$ where the model breaks down, and so identifying numerically the threshold value $\theta=\theta_{\text {clog }}$ for which clogging first occurs with any accuracy is problematic.

Nonetheless, we observe that only a restricted range of values $\theta_{\text {crit }}<\theta<\theta_{\text {clog }}$ provide a stable partially-clogged steady state solution. For $n>1$ we have $\theta_{\text {crit }}=0$, but for the particular choice $n=1$ we have $\theta_{\text {crit }}>0$ and the range is more tightly constrained. The clogging threshold $\theta_{\text {clog }}$ is an increasing function of shear exponent $m$. These characteristics provide scope for comparison with experiment in order to identify realistic parameter ranges for $\theta, n$, and $m$ more closely.

Simulations with a variety of nonuniform choices of initial condition $s_{0}(x)$ suggest also that steady states with maximum biofilm thickness away from the inflow (or rather the upstream extent of the initial condition) either do not exist or are only realisable under very specific conditions. Figure 5 shows biofilm thickness profiles at various times for a typical nonuniform solution. Maximum biofilm thickness migrates upstream to the limit of compact support of the initial condition: biofilm growth rates are higher upstream due to the effect of the longitudinal nutrient gradient being stronger than the variation in detachment rate. Thus under the current model a sharp interface develops at the upstream end of the biofilm, and we note that the assumption $s_{x} \ll 1$ is no longer met so that a fully $2 \mathrm{D}$ model of the 
biofilm and pore flow would be more appropriate locally in this region. In this case, one would still expect maximum biofilm thickness to be located close to the upstream end, but the local biofilm profile would depend on biofilm flow under growth-generated pressures and the 2-dimensional nutrient concentration distribution in both the fluid and the biofilm.

\section{Conclusions}

We describe a model of biofilm growth in a long 2D pore which aims to be a paradigm for studying bioclogging of porous media such as soil. Transport of nutrient from the inflow provides for a competition between nutrient supply, biofilm growth and detachment in which biofilm growth constricts the flow and can thereby restrict or block nutrient supply. We address the question of whether biofilm growth can proceed to the extent that it fully blocks the pore, or whether a partially-clogged steady state exists. Previous 1D theoretical models $[1,11]$ have identified conditions under which trivial and nontrivial steady states occur and have suggested that full clogging does not arise. The present extension to $2 \mathrm{D}$ provides a coupling of growth along the pore length via nutrient supply from the pore flow, which is not present in the $1 \mathrm{D}$ models.

The model comprises a uniform long 2D channel in which biofilm grows on the walls in response to nutrient supply and subject to erosion-like detachment. Following previous work [28], the biofilm is modelled as a two phase fluid based on polymer solution theory, and its large aspect ratio motivates a quasi-1D simplification. Pore flow is treated as thin film flow driven either by a fixed flow rate, or by a fixed pressure drop between pore ends. Nutrient is transported in the pore flow by advection and diffusion, and in the biofilm by diffusion and uptake. The lack of conclusive data on the functional form of the biofilm detachment leads us to investigate its dependence on biofilm thickness and fluid shear stress and the impact of this on biofilm distribution and clogging along the pore.

The model largely confirms the core results of previous one dimensional studies $[1,11]$ concerning the existence and stability of steady state biofilm thicknesses. In particular, physically reasonable solutions demand that detachment have an increasing dependence on shear stress and be at least linearly dependent on local biofilm thickness. A partially-clogged steady state can exist for sufficient inflow nutrient levels only within a limited range of detachment parameters. These characteristics provide scope for comparison with experiment in order to identify realistic detachment descriptions. Unlike the 1D models, our 2D model suggests that under conditions of flow driven by a fixed pressure drop, the longitudinal coupling of nutrient status and flow rate results in solutions where the biofilm may clog the full channel width. In the approach to a steady state, the biofilm thickness is always greatest at the inflow end of the channel due to nutrient depletion in the flow downstream. This stands in contrast to the well-mixed one-dimensional bioreactor model [1], in which nutrient limitation due to constricted flow provides a partially-clogged stable steady state and prevents full clogging.

The physical basis for a linear-or-greater dependence of detachment on biofilm thickness is unclear from a theoretical perspective. Potential explanations include stratification of biofilm cohesion $[7,15]$ and a probability distribution of detachment of finite sized biofilm particles [20]. While we have modelled a surface detachment term, we note that Morgenroth [13] 
identifies an alternative 'volumetric detachment' approach treating detachment as applying uniformly through the biofilm thickness (akin to an additional intrinsic decay term), though this approach likewise suffers a lack of connection to specific physical mechanism. Further experimental examination of biofilm detachment processes is therefore required.

The aim of our model is to provide a basis for upscaling by considering bundles and networks of such channels to describe biofilm growth in a porous medium. We would then envisage comparison of the upscaled model to lab-scale experiments on biofilm growth in simple artificial porous media [cf. 16] before application to soil column experiments.

\section{Acknowledgements}

This publication has emanated from research conducted with the financial support of Science Foundation Ireland under grant SFI/09/IN.1/I2645. S.B.G.O'B. acknowledges financial support from Science Foundation Ireland grant SFI/12/IA/1683.

\section{References}

[1] F. Abbas, R Sudarsan, and H. J. Eberl. Longtime behavior of one-dimensional biofilm models with shear dependent detachment rates. Math. Biosci. Eng., 9(2):215-239, 2012.

[2] P Baveye, P Vandevivere, B L Hoyle, P C DeLeo, and D Sanchez de Lozada. Environmental impact and mechanisms of the biological clogging of saturated soils and aquifer materials. Crit. Rev. Environ. Sci. Technol., 28(2):123-191, 1998.

[3] J Bear. Hydraulics of groundwater. McGraw-Hill, 1979.

[4] T. P. Clement, B. S. Hooker, and R. S. Skeen. Macroscopic models for predicting changes in saturated porous media properties caused by microbial growth. Ground Water, 34(5):934-942, 1996.

[5] J. W. Costerton, Z. Lewandowski, D. E. Caldwell, D. R. Korber, and H. M. Lappin-Scott. Microbial biofilms. Annu. Rev. Microbiol., 49:711-745, 1995.

[6] A. B. Cunningham, R. R. Sharp, R. Hiebert, and G. James. Subsurface biofilm barriers for the containment and remediation of contaminated groundwater. Bioremediation J., 7:151-164, 2003.

[7] N. Derlon, A. Massé, R. Escudié, N. Bernet, and E. Paul. Stratification in the cohesion of biofilms grown under various environmental conditions. Water Res., 42(8):2102-2110, 2008.

[8] R. Duddu, D. L. Chopp, and B. Moran. A two-dimensional continuum model of biofilm growth incorporating fluid flow and shear stress based detachment. Biotech. Bioeng., 103(1):92-104, 2009.

[9] H. J. Dupin, P. K. Kitanidis, and P. L. McCarty. Pore-scale modeling of biological clogging due to aggregate expansion: A material mechanics approach. Water Resour. Res., 37(12):2965-2979, 2001.

[10] C. C. Ezeuko, A. Sen, A. Grigoryan, and I. D. Gates. Pore-network modeling of biofilm evolution in porous media. Biotechnol. Bioeng., 108(10):2413-2423, 2011. doi: 10.1002/bit.23183.

[11] I. Klapper. Productivity and equilibrium in simple biofilm models. Bull. Math. Biol., 74(12):2917-2934, 2012.

[12] R Kommedal and R Bakke. Modeling Pseudomonas aeruginosa biofilm detachment. HiT Working Paper 3/2003, Høgskolen i Telemark, 2003. 
[13] E. Morgenroth. Detachment: an often-overlooked phenomenon in biofilm research and modelling. In S. Wuertz, P. L. Bishop, and P. A. Wilderer, editors, Biofilms in wastewater treatment: an interdisciplinary approach. IWA Publishing, 2003.

[14] T. R. R. Pintelon, C. Picioreanu, M. C. M. van Loosdrecht, and M. L. Johns. The effect of biofilm permeability on bio-clogging of porous media. Biotechnol. Bioeng., 109(4):1031-1042, 2012.

[15] M Ras, D Lefebvre, N Derlon, J Hamelin, N Bernet, E Paul, and E Girbal-Neuhauser. Distribution and hydrophobic properties of Extracellular Polymeric Substances in biofilms in relation towards cohesion. J. Biotechnol., 2013.

[16] H. C. Rees, S. E. Oswald, S. A. Banwart, R. W. Pickup, and D. N. Lerner. Biodegradation processes in a laboratory-scale groundwater contaminant plume assessed by fluorescence imaging and microbial analysis. Appl. Environ. Microbiol., 73(12):3865-3876, 2007.

[17] B. E. Rittmann. The effect of shear stress on biofilm loss rate. Biotechnol. Bioeng., 24(2):501-506, Feb 1982.

[18] A. Rochex, A. Massé, R. Escudié, J.-J. Godon, and N. Bernet. Influence of abrasion on biofilm detachment: evidence for stratification of the biofilm. J. Ind. Microbiol. Biotechnol., 36(3):467-470, 2009.

[19] R. Rusconi, S. Lecuyer, N. Autrusson, L. Guglielmini, and H. A. Stone. Secondary flow as a mechanism for the formation of biofilm streamers. Biophysical J., 100(6):1392-1399, 2011.

[20] P. S. Stewart. A model for biofilm detachment. Biotechnol. Bioeng., 41:111-117, 1993.

[21] T. L. Stewart and D. S. Kim. Modeling of biomass-plug development and propagation in porous media. Biochem Eng J, 17(2):107-119, 2004.

[22] S. W. Taylor, P. Milly, and P. R. Jaffé. Biofilm growth and the related changes in the physical properties of a porous medium 2. permeability. Water. Resour. Res., 26(9):2161-2169, 1990.

[23] M. Thullner, J. Zeyer, and W. Kinzelbach. Influence of microbial growth on hydraulic properties of pore networks. Transp. Porous. Media, 49:99-122, 2002.

[24] T. L. van Noorden, I. S. Pop, A. Ebigbo, and R. Helmig. An upscaled model for biofilm growth in a thin strip. Water Resour. Res., 46:W06505, 2010.

[25] S. J. Vogt, A. B. Sanderlin, J. D. Seymour, and S. L. Codd. Permeability of a growing biofilm in a porous media fluid flow analyzed by magnetic resonance displacement-relaxation correlations. Biotechnol. Bioeng., 110(5):1366-1375, 2012.

[26] O. Wanner and W. Gujer. A multispecies biofilm model. Biotechnol. Bioeng., 28:314-328, 1986.

[27] W. B. Whitman, D. C. Coleman, and W. J. Wiebe. Prokaryotes: the unseen majority. PNAS, 95(12): 6578-6583, 1998.

[28] H. F. Winstanley, M. Chapwanya, A. C. Fowler, and M. J. McGuinness. A polymer-solvent model for biofilm growth. Proc. R. Soc. A, 467:1449-1467, 2011. 